\title{
CLINICOPATHOLOGICAL CASE CONFERENCE
}

\section{Progressive dementia and gait disorder in a 78 year old woman}

\author{
Michele Tagliati, Daniel P Perl, Burton Drayer, C Warren Olanow
}

\section{Case presentation}

This 78 year old right handed white woman had a medical history significant for a cerebellar tumour, removed at age 12 by Dr Harvey Cushing. Reportedly, this was an astrocytoma and the removal was successful. Her balance was never perfect, but she was able to walk unassisted. She lived at home with her husband and had two children who are in good health.

The patient was essentially well until November 1995 when on a trip to Israel she became rather tired and had increased difficulty in walking. On her return from this trip, she complained of fatigue, lethargy, dizziness, and progressive memory loss. In January 1996 she developed bilateral hand tremor, evident both at rest and during action. Her primary care physician made a diagnosis of Parkinson's disease and treated her with levodopa and amantadine, with only temporary benefit. After an increase in the dose of levodopa, she experienced visual hallucinations and worsening tremor, which resolved on discontinuation of the drug.

At the end of March 1996, she had a transient episode in which she suddenly raised her right leg while seated and collapsed on the chair soon after. According to a witness, she lost consciousness for a few seconds. She was admitted to a community hospital where a neurological evaluation was unremarkable aside from a striking memory loss. Diagnostic investigations including head CT, EEG, carotid Doppler, and MRA were essentially negative and she was discharged with a diagnosis of transient ischaemic attack. Since then her balance progressively worsened. Her recent memory and thought processes were poor. Confusion, fatigue, and sleepiness developed, with bradykinesia, urinary urgency, and frequency. She also complained of continuous dizziness. This was present when she was lying, sitting, or standing. She felt as if the room was moving around her.

On our initial neurological evaluation in May 1996, she was alert and pleasant, but unable to identify the place or the year. She was able to give coherent responses but the richness of her speech was decreased. Her speech was of normal rhythm. She could spell WORLD backwards. Immediate recall and short term memory were very poor. She knew that the current president was Clinton, but could not identify any president before that. Calculations, although impaired, were considerably better than her short term memory. Pupils were normal and reactive. Fundi were normal. Visual fields were intact, with 20/25 visual acuity. She had some difficulty in ocular pursuit to the right and nystagmus with slow phase to the right. There were no carotid bruits. Motor and sensory testing was within normal limits except for mild distal symmetric vibratory loss in the lower limbs. Rapid alternating movements of the hands were intact. She had bilateral terminal tremor on finger to nose, somewhat worse on the left side. She could not stand with feet together and eyes closed, but could stand with support with eyes opened. Her gait was broad based, she fell to either side and she could not tandem walk. A brain MRI was reportedly normal, aside from mild dilatation of the lateral ventricles and prominence of the cerebral sulci and sylvian fissures.

She was admitted to the Neurology Service on August 1996 to undergo a lumbar puncture with drainage of more than $30 \mathrm{ml} \mathrm{CSF}$. Opening pressure was $15 \mathrm{~cm} \mathrm{H}_{2} \mathrm{O}$. Results of the routine laboratory studies on her blood and CSF were within normal limits. After the large volume tap, there was no change in her neurological condition. She was discharged 1 day later with a diagnostic impression of early Alzheimer's disease superimposed on chronic cerebellar dysfunction.

By December 1996, she was unable to write, read, or initiate conversation. Gait was unsteady and the left side was described as slightly weaker than the right. A mild intention tremor was noted bilaterally. A repeat MRI of the brain, with and without contrast infusion, showed dilation of the ventricular system, sulcal prominence without evidence of midline shift or mass effect, and postsurgical changes of the cerebellar hemispheres. Signal hyperintensity on T2 images involving the left temporal lobe and both thalami was also evident. A diagnostic impression of gait disorder secondary to multi-infarct dementia was formulated.

Her clinical condition gradually worsened and on May 1997 she was sleeping most of the day. Mini mental state evaluation gave a score of $5 / 30$. She had frontal release signs, bilateral Babinski's sign, unsteady gait, and intermittent sphincter incontinence. She was able to take only one small step with assistance and was 
wheelchair bound. Medications included Inderal for tremor, ritalin for hypersomnia, zoloft for depression, and aspirin for CVA prophylaxis. On 14 May 1997, she experienced an episode of sudden "collapse" with incontinence of urine and stool. An EEG showed a poorly organised, symmetric theta rhythm with additional delta slowing, without evidence of epileptic activity. She gradually deteriorated and died at home in June 1997. A necropsy was obtained.

\section{Differential diagnosis}

Dr C Warren Olanow

Let me go through some of the features that characterise this rather complicated case. The patient was a 78 year old woman with a clinical course that seems to have been dominated by dementia. I don't think that the tumour that she was operated on for over 60 years ago has anything to do with the current illness. She had some slight residual disturbance of balance, but she was otherwise intact.

The current problem began in November 1995 with non-specific weakness and fatigue, and progressive memory loss. In a 78 year old person who presents with progressive memory loss, the first cause one would consider is Alzheimer's disease. ${ }^{1}$ Vascular dementia is also relatively common, but a vast majority of patients with vascular dementia also have Alzheimer's disease changes. ${ }^{2}$ In January of 1996 she was diagnosed with Parkinson's disease. She had resting and action tremor, worsening gait dysfunction, and probably some rigidity. ${ }^{3}$ At that point, one may consider that there was involvement of the basal ganglia and the cerebral hemispheres, at least the hippocampal region, and that we might be dealing with a combination of Alzheimer's disease and parkinsonism or Lewy body disease. ${ }^{4}$

In March 1996, added to the problem is an episode in which she experienced a sudden movement of the right leg followed by transient loss of consciousness. Despite a provisional diagnosis of transient ischaemic attack, this episode should probably be considered a seizure and suggests the involvement of the cerebral cortex. Patients with Parkinson's disease not only do not have seizures, but are relatively protected from having seizures. This may be due to the fact that the globus pallidus and the substantia nigra pars reticulata flood the brainstem with inhibitory stimuli and tend to turn off the structures that promote seizures. We did a study several years ago and noticed that in patients with epilepsy who develop Parkinson's disease seizures become easier to control. On the other hand, patients with Alzheimer's disease or other types of dementing illnesses have a tendency to develop seizures. ${ }^{5}$ But in this setting, they tend to occur rather late in the course of the illness and we have only 6 months of clinical progression in this patient.

The patient then began to notice that her balance was progressively worsening. This may be due to her old cerebellar problem, or to basal ganglia dysfunction with postural instability as she complained of worsening bradyki- nesia. A new complaint of dizziness suggests involvement of the brainstem, or cerebellar or vestibular system. In May of 1996 she had substantially deteriorated, although she retained some elements of memory. Difficulties with ocular pursuit, and a broad based gait were seen at this point. This is not what you see in Parkinson's disease, in which there is an apraxia of gait, with difficulty initiating gait. When you see a broad based gait, it suggests that there is a problem with cerebellar function or a dysfunction of the input to the cerebellum. It is difficult to distinguish in this case what may be an old cerebellar problem aggravated by the stress and strain of the current illness from what may be a new cerebellar impairment, in addition to cortical, basal ganglia, and brainstem involvement. The case raises the notion of diffuse involvement of the CNS.

She was brought to the hospital in August to rule out the "obligatory" diagnosis of normal pressure hydrocephalus. Patients with true normal pressure hydrocephalus develop gait disturbance and urinary incontinence before they become demented and have characteristic imaging features. ${ }^{6}$ If they do not fit in this typical picture, they do not benefit from shunting and probably do not have normal pressure hydrocephalus. Dementia was clearly the dominant problem in this case and it exceeded and anteceded the development of the other problems. Therefore, I do not think that this patient had normal pressure hydrocephalus. In fact, she was not treated for it and was discharged with a diagnosis of Alzheimer's disease.

The concerning detail is that the dementia progressed over a relatively short period of time, and by December-only 1 year after the onset of her symptoms - she was unable to write, unable to read, and unable to initiate a conversation. This degree of dementia is far too profound in comparison to what you would expect to see with conventional Alzheimer's disease. This raises the notion of other conditions, such as prion disease. Prion disease has been in the news a great deal recently because of the epidemic of bovine spongiform encephalopathy that has plagued Great Britain. Many cases of atypical prion disease have been recently described, in which brainstem, cerebellum, and thalamus are initially involved and dementia develops later in the course of the illness. ${ }^{7}$ Most of these patients die about 6 months after the onset of their illness, but longer patterns of evolution have been described in which patients survive for 12 to 18 months or longer. Whereas Alzheimer's disease, Parkinson's disease, and Lewy body disease would be unlikely to involve so many CNS structures, prion disease has the capacity to affect a larger number of structures and to rapidly evolve-in fact even more rapidly than we see in this case. In addition to all of the above, she developed a bilateral Babinski's response suggesting the involvement of the corticospinal tracts. She rapidly continued to deteriorate in all capacities and eventually died in June 1997, after a total clinical course of 18 months. 
At this point it is opportune to review the brain MRIs, because they show some interesting findings. Dr Drayer, can we review the neuroimaging studies?

Dr Burton Drayer

In the initial brain MRI study, performed in May 1996, there are clearly signal abnormalities on the T1 and T2 images in the left medial temporal lobe (fig 1), although this study was reported as normal. In addition, there are high signal abnormalities in the midbrain (fig $1 \mathrm{C}$ ) and thalamus bilaterally (fig $1 \mathrm{D}$ ). Some gyral swelling and subtle signal abnormality in the gyral region as well seem to suggest a more generalised process (fig $1 \mathrm{E}$ ), and can explain the clinical occurrence of seizures. All these areas of abnormal signal do not enhance. The cerebellum shows the old postsurgical abnormality (fig $1 \mathrm{~F}$ ) and the ventricles are enlarged.

A repeat study in October 1996 shows the same signal abnormalities in the thalamus, midbrain, and cerebral grey matter with very little change compared with the study performed 6 months earlier. Incidentally, MRA shows a very small right vertebral artery that could be a normal variant or secondary to atherosclerotic changes.

In summary, we have signal hyperintensity in the thalamus bilaterally, the midbrain, the left medial temporal lobe, and in the gyral cerebral cortex. The primary differential diagnosis of a picture that does not dramatically change over 6 months and does not enhance would be prion encephalopathy. Creutzfeld-Jakob disease classically presents high signal abnormalities in the putamen, but thalamic abnormalities and gyral swelling have been reported. ${ }^{8}$ Interestingly, another form of spongiform encephalopathy, kuru, typically involves the limbic system and the thalamus.

Another definite diagnostic possibility would be gliomatosis cerebri. ${ }^{9}$ This diagnosis is often very difficult clinically and generally presents with multifocal, extensive brain abnormalities in association with EEG slow wave activity. Limbic encephalopathy is a known clinical presentation. Finally, and less likely, slow virus encephalitides, such as subacute sclerosing panencephalitis might present with this type of MRI abnormality.

\section{Radiological diagnosis}

(1) Prion disease

(2) Gliomatosis cerebri

\section{Dr C Warren Olanow}

These films show something that you would not expect to see in Parkinson's disease, Alzheimer's disease, or Lewy body disease. As Dr Drayer mentioned, prion disease can show high signal abnormalities in the basal ganglia and the question is whether or not the abnormal signal described in the thalamus fits this picture. Recent studies have shown that the thalamus is more often involved in prion disease than was previously thought. Fatal familial insomnia seems particularly prone to involve the thalamus, ${ }^{10}$ but I am not aware of reports describing this particular pattern on
MRI. In addition, this patient presented with hypersomnia and not insomnia. What is striking in this case is the simultaneous involvement of the thalami, the left temporal lobe, and the cortical grey matter in a rapidly progressive neurological disease with dementia, gait disturbance, seizures, and death in about 1 year. Viral (herpes) encephalitis can involve the CNS diffusely and can explain the cortical swelling seen in this case, but it usually presents with a raging course. Moreover, the normal CSF analysis makes the diagnosis of encephalitis highly unlikely.

I think we have to give serious consideration to the idea that this is a neoplastic process and in particular gliomatosis cerebri. Gliomatosis cerebri tends to involve multiple CNS structures including the brainstem, the thalamus, the cerebral lobes, and affects both the grey and white matter. It could cause seizures and progress rapidly with features of dementia, corticospinal tract abnormalities, dizziness, and headache. ${ }^{11}$ There have been about 160 cases of gliomatosis cerebri described in the literature to date. The most frequent clinical features in gliomatosis cerebri are corticospinal tract findings (58\%), dementia (44\%), and spinal-cerebellar deficits (33\%). On review of the literature, the striking point is that every case presents in a unique way. There is no pattern that characterises gliomatosis cerebri, its presentation being a function of whatever CNS structure happens to be involved. Gliomatosis cerebri abnormalities are usually not detected by CT. Brain MRI typically shows bilateral and diffuse infiltration of midline structures. In 22 cases, ${ }^{12}$ there was involvement of the basal ganglia and thalamus (17/22), corpus callosum $(12 / 22)$, brainstem (3/22), and cerebellum $(2 / 22)$. Changes are only noted in T2 weighted scans and do not tend to enhance. The diagnosis usually cannot be made clinically but can be suspected based on the progression of symptoms and signs and the MRI pattern. Ultimately, a brain biopsy or necropsy is needed to confirm the diagnosis of gliomatosis cerebri. Total radiation may improve the median survival, which ranges from 6 to 9 months in untreated cases. ${ }^{13}$

In summary, the diffuse involvement of multiple CNS regions including the cortex, hemispheres, basal ganglia, thalamus, brainstem, and possibly the cerebellum with evidence of diffuse swelling on MRI are most consistent with the diagnosis of gliomatosis cerebri. Whereas parkinsonism is a most unusual presentation of gliomatosis cerebri, dementia is a common manifestation, and I think that the diagnosis of gliomatosis cerebri is what best explains what happened to this patient.

\section{Clinical diagnosis}

Alzheimer's disease; parkinsonism; possible prion disease

\section{Dr Olanow's diagnosis}

Gliomatosis cerebri 

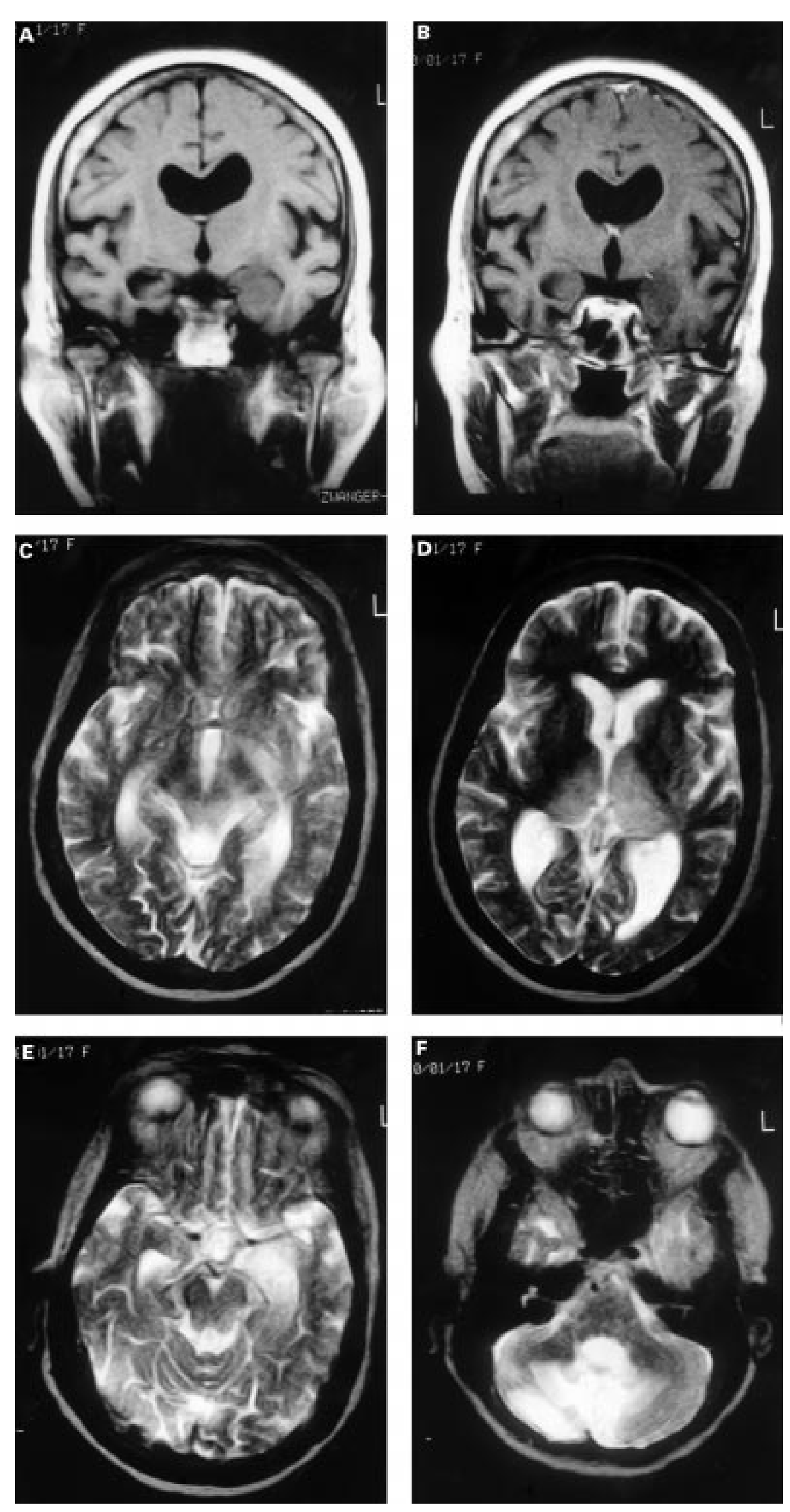

Figure 1 Initial brain

May 1996. (A,B)

Non-enhanced and

enhanced T1 coronal
images with decreased

signal and swelling of the

left temporal lobe and no

abnormal enhancement.

$(C, D, E)$ Signal

hyperintensity in the left

temporal, midbrain,

bilaterally in the thalamus,

and subtle changes in the

cortex on T2 axial images.

(F) Surgical changes in
cerebellum and signal

hyperintensity in left

temporal lobe on T2 axial

images.

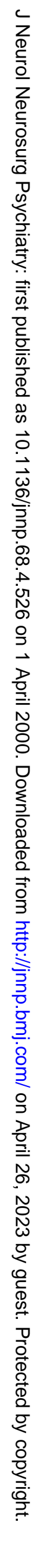




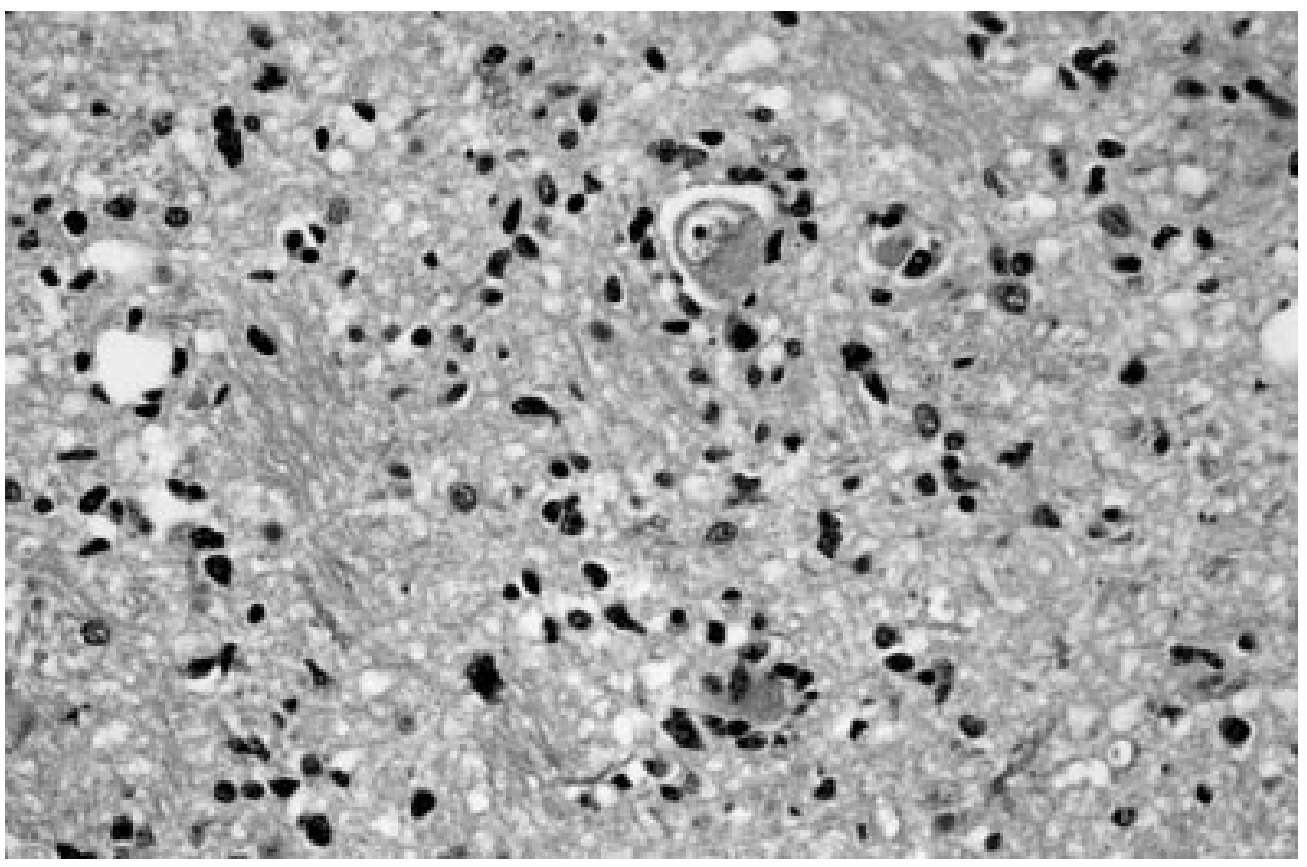

Figure 2 Photomicrograph, original magnification $\times 240$, haematoxylin and eosin. Appearance of the dorsal raphe nucleus showing infiltration of pleomorphic, atypical astrocytes with residual intact neurons remaining.

\section{Pathological discussion \\ Dr Daniel Perl}

We received this brain from an outside hospital. This patient was enrolled in our Alzheimer's disease brain bank programme, and following our standard dissection protocol, only the right side of the brain was analysed. The external aspect of the brain showed no abnormality. The medial aspect disclosed only non-specific dilatation of the lateral ventricle and some changes in the cerebellar area where Dr Cushing had done his surgery. There was scarring in the midline cerebellar vermis. There was no residual tumour in the fourth ventricle. Sixty nine years after surgery, this area looked remarkably clean. The substantia nigra and locus coeruleus looked normally pigmented, without gross abnormalities. Similarly, no obvious gross abnormality could be seen on coronal sections of the cerebral hemispheres. A Bielchowsky stain of the hippocampus showed no neurofibrillary tangles and no senile plaques. There were diffuse plaques in the cortex, consistent with age related changes in a 78 year old woman, but there was no evidence of Alzheimer's disease. Further, there were no spongiform changes indicative of a prion related disease.

In the pons, there was a suggestion of hypercellularity even at low power. The cerebellar peduncles were remarkably hypercellular, with diffuse infiltration of cells. At higher power, these cells had very variable appearance with small pyknotic nuclei alternating with large atypical and frankly neoplastic nuclei scattered all through the tissue. The locus coeruleus was normally pigmented but diffusely infiltrated by numerous, pleomorphic cells that had the appearance of neoplastic astrocytes.

In the midbrain, the substantia nigra was normally pigmented. No Lewy bodies were seen, therefore excluding the diagnosis of Par- kinson's disease. However, the extensive cellularity was again evident throughout. The oculomotor nuclei and the raphe neurons were surrounded by the same extensive cellularity, with atypical, neoplastic features at higher power (fig 2).

The thalamus was also quite hypercellular, without evidence of abnormal vasculature. The hippocampus did not show diffuse infiltration, although at higher power we were able to see individual atypical astrocytes, likely part of the same neoplastic process. Atypical astrocytic cells, with clear neoplastic features diffusely infiltrated the amygdala and inferior temporal lobe. The cerebral cortex looked normal at low power, but looking with care it was possible to see individual atypical astrocytic nuclei scattered throughout this tissue. The cortex was therefore involved, but not as extensively as we have seen in some other areas. The putamen looked similarly infiltrated.The abnormal cells were astrocytes; they stained positively with glial fibrillary acidic protein, and the pattern of diffuse infiltration was consistent with the diagnosis of gliomatosis cerebri.

Gliomatosis cerebri is a disorder that was first described by Samuel Nevin in 1938 as a diffuse proliferation of pleomorphic, neoplastic glial cells widely distributed throughout the CNS. ${ }^{14}$ Two types of gliomatosis cerebri are recognised ${ }^{15}$; one in which there is no underlying glioblastoma epicentre and the other in which there is an identifiable focal neoplastic nidus with extensive dissemination of neoplastic astrocytes throughout the CNS, far exceeding what is typically seen in glioblastoma. This case seems to belong to the first type.

Pathological diagnosis

Gliomatosis cerebri 
We thank the patient's husband for his compassionate help. We are grateful to Drs Bernard Cohen and Stanley Tuhrim for providing clinical information. Dr Seymour Gendelman offere insightful revision of the manuscript. This work was supported in part by 1RO1NS33772-04, 1RO1NS34796-01A1 and Rounds, The Mount Sinai Hospital, New York NY, October 26, 1998.

1 Geldmacher DS, Whitehouse PJ. Evaluation of dementia. $N$ Engl F Med 1996;335:330-6.

2 Nyenhuis DL, Gorelick PB. Vascular dementia: a contemporary review of epidemiology, diagnosis, prevention, and treatment. $\mathcal{F}$ Am Geriatr Soc 1998;46:1437-48.

3 Gelb DJ, Oliver E, Gilman S. Diagnostic criteria for Parkinson's disease. Arch Neurol 1999;56:33-9.

4 Gomez-Tortosa E, Ingraham $\mathrm{AO}$, Irizarry $\mathrm{MC}$, et al. Dementia with Lewy bodies. I Am Geriatr Soc 1998;46 $1449-58$

5 McAreavey MJ, Ballinger BR, Fenton GW. Epileptic seizures in elderly patients with dementia. Epilepsia 1992;33:657-60.

6 Pickard JD. Normal pressure hydrocephalus. In: M Swash, J Oxbury, eds. Clinical neurology. New York: Churchill Oxbury, eds. Clinical neur
Livingston, 1991:151-64.
7 Johnson RT, Gibbs CJ Jr. Creutzfeld-Jacob disease and related transmissible spongiform encephalopathies. $N$ Engl f Med 1998;339:1994-2004

8 Bahn MM, Parchi P. Abnormal diffusion-weighted magnetic resonance images in Creutzfeldt-Jakob disease. Arch Neurol 1999;56:577-83.

9 Spagnoli MV, Grossman RI, Packer RJ, et al. Magnetic resonance imaging determination of gliomatosis cerebri. Neuronance imaging determinatio

10 Lugaresi E, Tobler I, Gambetti P, et al. The pathophysiology of fatal familial insomnia. Brain Pathol 1998;8:521-6.

11 Artigas J, Cervos-Navarro J, Iglesias JR, et al. Gliomatosis cerebri: clinical and histological findings. Clin Neuropathol 1985; $4: 135-48$

12 Del Carpio-O'Donovan R, Korah I, Salazar A, et al. Gliomatosis cerebri. Radiology 1996;198:831-5.

13 Kim DG, Yang HJ, Park IA, et al. Gliomatosis cerebri: clinical features, treatment, and prognosis. Acta Neurochir (Wien) 1998;140:755-62.

14 Nevin S. Gliomatosis cerebri. Brain 1938;61:170-191.

15 Russell DS, Rubinstein LJ. Tumours of central neuroepithelial origin. In: Russell DS, Rubinstein LJ, eds. Pathology of tumours of the nervous system, 5th ed. Baltimore: Williams and Wilkins, 1989;146-8. 